\title{
Real-World Experience with Nivolumab in Metastatic Renal Cell Carcinoma Patients Who Have Progressed on Prior Therapies: A Single-Center Study from India
}

\author{
Amit Rauthan ${ }^{10} \quad$ Nitin Yashas Murthy ${ }^{10}$ \\ Shabber S. Zaveri
}

\begin{abstract}
Address for correspondence Amit Rauthan, MBBS MD (Internal Medicine) DM (Medical Oncology), Department of Medical Oncology, Manipal Hospitals, Old Airport Road, Bangalore, Karnataka 560017, India (e-mail: amitrauthan@yahoo.com).
\end{abstract}

South Asian J Cancer 2022;11(2):133-139.

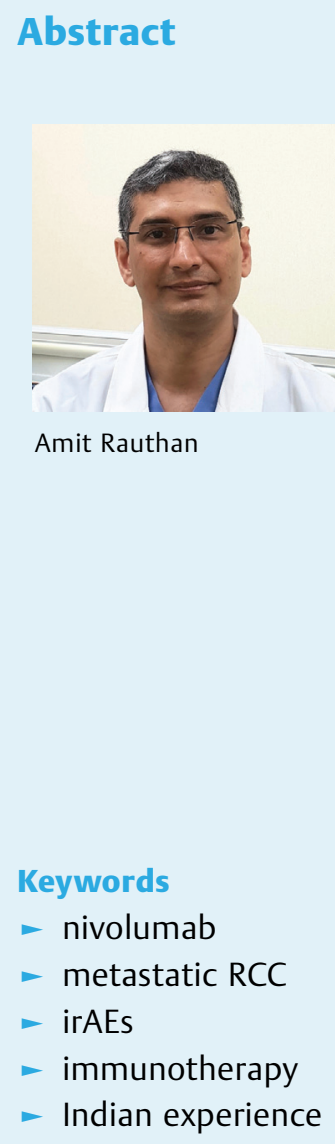

Introduction Nivolumab monotherapy is approved for the treatment of metastatic renal cell carcinoma ( $\mathrm{mRCC}$ ) patients who have progressed on prior therapies based on the pivotal Checkmate-025 trial. There is limited literature on the efficacy and safety profile of usage of nivolumab in the treatment of mRCC in India in a real-world setting. Methods A retrospective analysis was performed of patients who received nivolumab monotherapy for mRCC after having progressed on prior therapies. Tumor response was graded according to RECIST v1.1 and Kaplan-Meier survival analysis was used to estimate progression-free survival (PFS) and overall survival (OS). Immune-related adverse events (irAEs) were documented and graded according to CTCAE v5.0.

Results Between 2016 and 2019, 35 patients received nivolumab for mRCC at our center after progression on prior therapies. A majority of the patients $(n=30,85.7 \%)$ received it in a second-line setting, and the remaining in the third line and beyond setting. Clear cell was the most common histology $(n=26,74.3 \%)$. There were 18 patients $(51.42 \%)$ who belonged to IMDC intermediate risk, while 17 (48.58\%) patients were at poor risk. The overall response rate was $60 \%$, with complete response (CR) in $11.4 \%$. Median duration of response was not reached among responders. Median PFS was 5 months (95\% confidence interval $[\mathrm{Cl}]$ : 3.06-6.93) and median OS was 26 months (95\% Cl: 1.90-50.09). Ongoing survival of 47, 42, 34, and 22 months was noted in four patients with CR, respectively. In our study, 23 patients (65.71\%) experienced any grade of irAE. Grade 3 irAEs was seen in four patients (11.42\%). Most common irAE was thyroid dysfunction seen in 12 patients (34.2\%). Treatment discontinuation due to irAEs occurred in three patients (8.57\%).

Conclusion Nivolumab showed good efficacy with high response rates and an OS comparable to the pivotal Checkmate- 025 trial. It was well tolerated with safety profile in terms of irAE consistent with those reported in literature.
DOI https://doi.org/10.1055/s-0041-1740373 ISSN 2278-330X

How to cite this article: Rauthan A, Murthy NY, Patil P, et al. RealWorld Experience with Nivolumab in Metastatic Renal Cell Carcinoma Patients Who Have Progressed on Prior Therapies: A SingleCenter Study from India South Asian J Cancer 2022;11(2): $133-139$. (c) 2022. MedIntel Services Pvt Ltd. All rights reserved.

This is an open access article published by Thieme under the terms of the Creative Commons Attribution-NonDerivative-NonCommercial-License, permitting copying and reproduction so long as the original work is given appropriate credit. Contents may not be used for commercial purposes, or adapted, remixed, transformed or built upon. (https://creativecommons.org/licenses/by-nc-nd/ 4.0/)

Thieme Medical and Scientific Publishers Pvt. Ltd., A-12, 2nd Floor, Sector 2, Noida-201301 UP, India 


\section{Introduction}

Renal cell carcinoma (RCC) refers to a heterogeneous group of cancers arising from renal tubular epithelial cells, and constitutes almost $90 \%$ of all primary renal neoplasm. ${ }^{1}$ In 2020 in India, there were 16,861 new kidney cancer cases contributing to $1.3 \%$ of new cancers. It ranked 22 nd in terms of mortality with 9,897 deaths accounting for $1.2 \%$ of mortality. ${ }^{2}$ Over the years, there has been a significant evolution in the treatment of metastatic renal cell carcinoma (mRCC). In the last decade, vascular endothelial growth factor tyrosine kinase inhibitors (VEGF TKIs) like sunitinib or pazopanib have become the standard first-line treatment approach in advanced RCC. ${ }^{3,4}$ Treatment of MRCC in second line included sequential usage of another VEGF TKI like axitinib or sorafenib or mammalian target of rapamycin pathway inhibitor everolimus. $^{5-7}$ But second-line treatment is still a challenge with limited therapeutic options, high morbidities, and limited survival.

Studies in the past showed that programmed death ligand 1 (PD-L1) expression is associated with a poor prognosis in RCC, probably because of its immunosuppressive function as the interaction between PD-1 expressed on activated T-cells and PD-ligand 1(PDL-1) or PD-L2 expressed on tumor cells usually results in inhibition of the cellular immune response, leading to escape of tumor cells from immunosurveillance. ${ }^{8}$ Hence, nivolumab, a fully human IgG4. PD-1 immune checkpoint inhibitor antibody that selectively blocks the interaction of PD-1 with PDL-1 and PDL-2, was studied in Phase- 1 and Phase2 studies. ${ }^{9-12}$ Subsequently, the publication of the landmark Phase-3 Checkmate 025 trial showed the superiority of nivolumab in comparison with everolimus in response and overall survival (OS) in previously treated patients of advanced/metastatic RCC. ${ }^{13}$ This has led to a revolutionary change in the treatment landscape of metastatic RCC with nivolumab becoming a standard second-line therapy in mRCC.

However, there is limited literature on the real-world efficacy and safety profile of nivolumab in metastatic RCC from India. Here, we report the response rates and survival along with the safety profile of patients with mRCC treated with nivolumab after having progressed on at least one prior therapy in a tertiary care center in India.

\section{Materials and Methods}

\section{Patient Selection}

Approval was obtained from the Institutional Ethics Review Board to perform a retrospective study at our center of patients with metastatic RCC who received treatment with nivolumab after having progressed on at least one line of prior therapy. Patients who were started on nivolumab between April 2016 and November 2019 were included in the study. The study follow-up period ended in April 2020.

\section{Study Methodology}

Demographic data, histological diagnosis, Eastern Cooperative Oncology Group performance status, prior therapies received, metastatic sites before initiation of nivolumab, laboratory parameters, and International mRCC Database Consortium (IMDC) risk stratification were obtained from the medical records.

Treatment protocol followed at our center consisted of intravenous administration of nivolumab at a dose of $3 \mathrm{mg} / \mathrm{kg}$ started at a frequency of 2-week interval. First response assessment with imaging was done post 4 cycles of therapy followed by periodic assessment at 3 monthly intervals.

Endpoints: 1) Response: Best response was graded as complete response (CR), partial response (PR), stable disease (SD), and progressive disease (PD) as per Response Evaluation Criteria in Solid Tumours (RECIST) v 1.1. Objective response rate was calculated as the proportion of patients who achieved a best response of CR or PR. 2) Survival: Progression-free survival (PFS) was defined as the time from first dose of nivolumab to first documented RECIST tumor progression or death from any cause. Information about patients who continued to receive treatment with nivolumab beyond RECIST defined progression in view of clinical benefit and absence of rapid progression was also documented. OS was defined as the time from first dose of nivolumab to death from any cause. Patients were censored on their last follow-up visit if the event of interest had not occurred by the end of study period. 3) Toxicity: Immunerelated adverse events (irAEs) were documented based on the clinical symptoms/laboratory/radiological parameters and were graded according to Common Terminology Criteria for Adverse Events (CTCAE) version 5.0. Treatment initiated for irAEs and rates of discontinuation of treatment due to irAEs were also noted.

\section{Statistical Analysis}

The statistical analysis was performed using SPSS 25. In descriptive statistics, the continuous variables are expressed as mean and standard deviation/median and interquartile range based on distribution of data. Categorical variables are expressed as frequency and percentage. Chi-squared test and Fisher's exact test were used to assess any association between categorical variables. Kaplan-Meier survival analysis was used to estimate survival events such as PFS and OS. Log rank test was used to calculate difference between survival events. All statistical analyses were tested at 95\% confidence interval ( $\mathrm{CI}$ ) and the $p$-value of $<0.05$ was considered as statistically significant.

\section{Results}

Between April 2016 and November 2019, 35 patients of mRCC who had progressed on prior therapy received nivolumab at our center. The median follow-up was 19 months. The median number of cycles of nivolumab received was 12 (interquartile range: $4-18$ ). The maximum number of cycles received by a patient was 37 .

\section{Baseline Characteristics}

A majority of the patients $(n=30,85.7 \%)$ patients received nivolumab as second-line treatment, while five received it in a third line and beyond setting. The median age of the study 
group was 58 years with majority of the patients being male $(n=28,80 \%)$. Clear cell carcinoma emerged as the most common histology in our study occurring in $74.3 \%$ of patients $(n=26)$. As per the IMDC risk classification, 18 patients (51.42\%) were stratified as IMDC intermediate risk group, and 17 (48.58\%) were stratified as IMDC poor risk. Lungs were the most common site of metastases seen in our study in 25 patients (71.4\%). Twenty-seven patients (77.14\%) had undergone nephrectomy prior to the initiation of nivolumab. These baseline characteristics are summarized in - Table 1.

\section{Objective Response and Duration of Response}

The objective response rate was $60 \%(n=21)$ in the entire study group. Best response of CR was noted in 4 (11.4\%) patients, PR in 17 patients (48.6\%), PD in 12 (34.3\%) patients, and SD in 2 patients (5.7\%). Among the 21 responders, the median duration of response was not reached. At the end of the study period, 16 patients (76.19\%) have an ongoing response with 11 patients $(52.38 \%$ ) having a response lasting for a year or more. Longest duration of ongoing response of 45 months, 40 months, 32 months, and 20 months was noted in four patients who continue to be in CR. Among the responders, progression of disease was noted in five patients, at 4 months in two patients, and in the rest at 3 months, 6 months, and 1 month, respectively.

\section{Progression-Free Survival and Treatment beyond Progression}

Median PFS in our study was 5 months (95\% CI: 3.06-6.93). The Kaplan-Meier estimate for 1-year PFS rate was $37.1 \%$. Among the 22 patients who had a RECIST-defined progression, 5 patients $(22.72 \%)$ were treated beyond progression in view of symptomatic improvement and clinical stability. Three of these 5 patients went onto achieve a best response of PR on further treatment with nivolumab and have an ongoing OS of 24 months, 20 months, and 19 months, respectively.

\section{Overall Survival}

The median OS in our study was 26.0 months (95\% CI: $1.90-$ 50.09) (-Fig. 1A). The four patients with CR have an ongoing survival of 47, 42, 34, and 22 months, respectively. Fifteen of the 17 deaths occurred before the end of 1 year since the initiation of treatment. In our study, the survival between responders and nonresponders was statistically significantly different (-Fig. 1B) with the median OS not reached in responders (95\% CI: NE-NE) and was only 4 months in nonresponders (95\% CI: 2.18-5.81) $(p<0.001)$. There was no statistically significant difference $(p=0.71)$ in the survival (-Fig. 1C) between the different IMDC risk groups as the median OS in the IMDC intermediate risk group was 20 months (95\% CI: NE-NE) and in the IMDC poor risk patients, it was 26 months (95\% CI: 1.83-50.16).

\section{Stoppage of Treatment in Responders}

At the end of the study period, out of the 21 responders, 9 responders are currently ongoing treatment with nivolumab. Among the 12 responders who stopped treatment, 4 patients
Table 1 Baseline demographic and clinical characteristics of patients receiving nivolumab in $\operatorname{mRCC}(n=35)$

\begin{tabular}{|c|c|}
\hline \multicolumn{2}{|c|}{ Line of usage of nivolumab in mRCC $(n, \%)$} \\
\hline Second line & $30(85.7 \%)$ \\
\hline Third line & $4(11.4 \%)$ \\
\hline Fourth line & $1(2.9 \%)$ \\
\hline \multicolumn{2}{|l|}{ Gender ( $n, \%)$} \\
\hline Male & $28(80 \%)$ \\
\hline Female & $7(20 \%)$ \\
\hline \multicolumn{2}{|l|}{ Age group ( $n, \%)$} \\
\hline $21-40$ years & $2(5.7 \%)$ \\
\hline $41-60$ years & $16(45.7 \%)$ \\
\hline $61-80$ years & $16(45.7 \%)$ \\
\hline$>80$ years & $1(2.9 \%)$ \\
\hline \multicolumn{2}{|l|}{ Histology $(n, \%)$} \\
\hline Clear cell & $26(74.3 \%)$ \\
\hline Clear cell + sarcomatoid changes & $4(11.4 \%)$ \\
\hline Collecting duct & $2(5.7 \%)$ \\
\hline Papillary & $2(5.7 \%)$ \\
\hline Chromophobe+ sarcomatoid changes & $1(2.9 \%)$ \\
\hline \multicolumn{2}{|l|}{ IMDC risk (n, \%) } \\
\hline Good risk & $0(0 \%)$ \\
\hline Intermediate risk & $18(51.4 \%)$ \\
\hline Poor risk & $17(48.6 \%)$ \\
\hline \multicolumn{2}{|l|}{ Prior nephrectomy $(n, \%)$} \\
\hline Yes & $27(77.1 \%)$ \\
\hline No & $8(22.9 \%)$ \\
\hline \multicolumn{2}{|l|}{ Sites of metastases $(n, \%)$} \\
\hline Lung & $25(71.4 \%)$ \\
\hline Bone & $12(34.3 \%)$ \\
\hline Lymph nodes & $12(34.3 \%)$ \\
\hline Liver & $5(14.3 \%)$ \\
\hline Brain & $5(14.3 \%)$ \\
\hline Peritoneal & $5(14.3 \%)$ \\
\hline Pleural & $4(11.4 \%)$ \\
\hline Adrenal & $3(8.6 \%)$ \\
\hline \multicolumn{2}{|c|}{ Prior systemic therapies in first-line setting $(n, \%)$} \\
\hline Sunitinib & $16(45.7 \%)$ \\
\hline Pazopanib & $15(42.9 \%)$ \\
\hline Chemotherapy & $3(8.5 \%)$ \\
\hline Sorafenib & $1(2.9 \%)$ \\
\hline \multicolumn{2}{|c|}{ Prior systemic therapies in second-line setting $(n, \%)$} \\
\hline Everolimus & $3(8.5 \%)$ \\
\hline Lenvatinib + everolimus & $1(2.9 \%)$ \\
\hline Axitinib & $1(2.9 \%)$ \\
\hline \multicolumn{2}{|c|}{ Prior systemic therapy in third-line setting $(n, \%)$} \\
\hline Sorafenib & $1(2.9 \%)$ \\
\hline
\end{tabular}

Abbreviation: mRCC, metastatic renal cell carcinoma. 


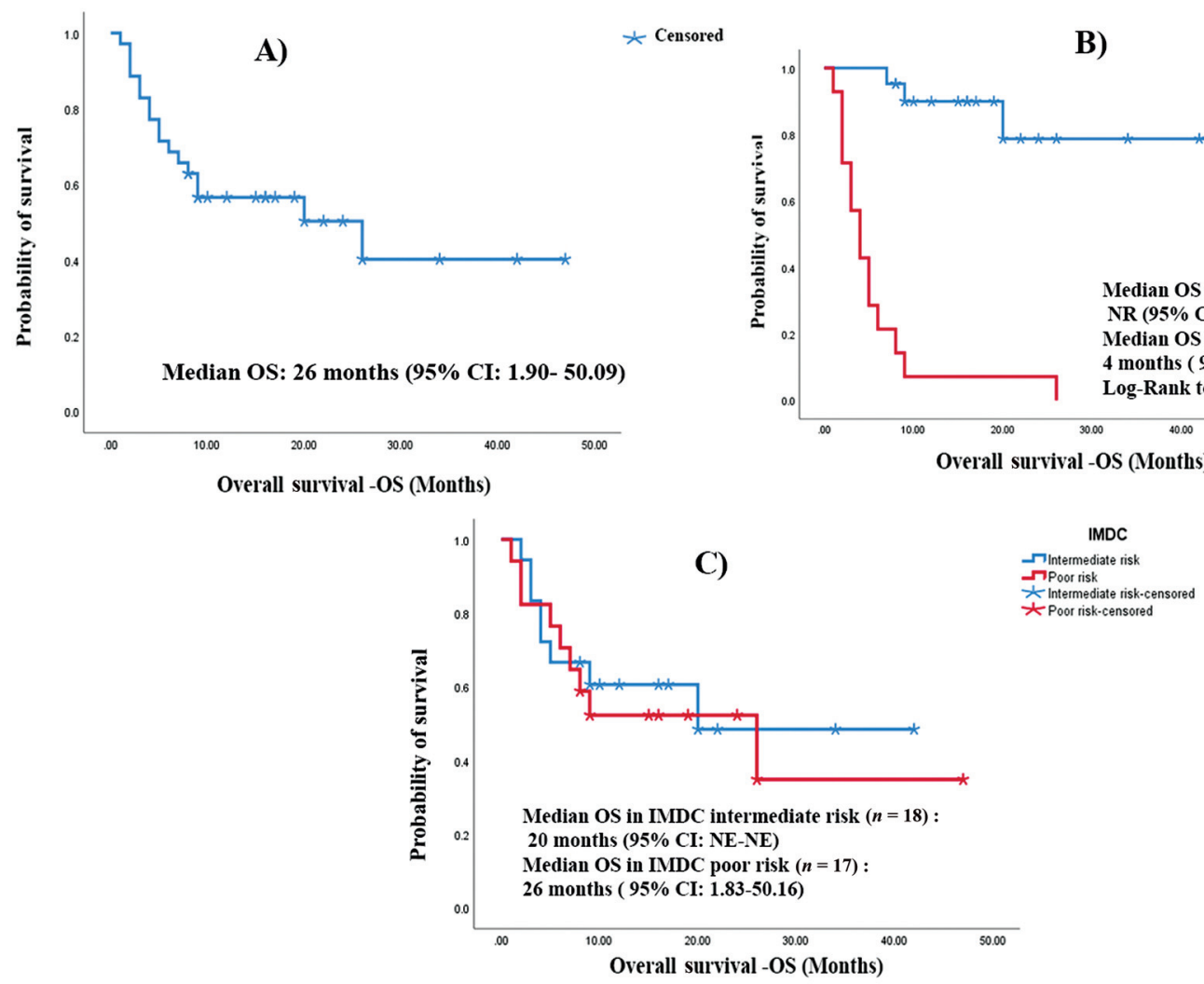

Fig. 1 Kaplan-Meier curve of (A) overall survival (OS) ( $n=35)$, (B) overall survival in responders v/s nonresponders, and (C) overall survival in IMDC intermediate risk v/s poor risk. $\mathrm{Cl}$, confidence interval.

stopped therapy due to progression and 1 stopped due to irAE. A further four patients who achieved CR stopped therapy after having received nivolumab for a duration of 1 year each. These patients are at 35 months, 30 months, 22 months, and 10 months of follow-up after stopping therapy and continue to be in CR. The remaining three patients stopped treatment at the lowest burden of disease and are on follow-up at 6 months, 8 months, and 5 months, respectively, after stoppage of treatment. They had received treatment for 6 months, 9 months, and 15 months, respectively.

\section{Safety Profile}

There were 23 patients (65.71\%) who experienced any grade of irAE. Grade1 to 2 irAEs were seen in 19 patients (54.28\%), while 4 patients (11.42\%) experienced Grade 3 irAEs ( - Table 2). The most common irAE was thyroid dysfunction seen in 12 patients (34.2\%) with a median onset of 10 weeks. Fatigue occurred in eight patients (22.8\%) with median onset being 6 weeks. Dermatological irAEs in form of skin rash/pruritus occurred in five patients (14.3\%) with median onset being 6 weeks. The most common Grade 3 irAE that

Table 2 irAEs in patients receiving nivolumab in mRCC $(n=35)$

\begin{tabular}{|c|c|c|c|}
\hline \multicolumn{2}{|l|}{ irAE } & Any grade (number of patients, \%) & Grade 3 (number of patients, \%) \\
\hline \multicolumn{2}{|l|}{ All irAE } & $23(65.7 \%)$ & $4(11.42 \%)$ \\
\hline \multirow{2}{*}{$\begin{array}{l}\text { Thyroid dysfunction } \\
(n=12)\end{array}$} & Hyperthyroidism & $4(11.42 \%)$ & $0(0 \%)$ \\
\hline & Hypothyroidism & $8(22.85 \%)$ & $0(0 \%)$ \\
\hline \multicolumn{2}{|l|}{ Fatigue $(n=8)$} & $8(22.85 \%)$ & $0(0 \%)$ \\
\hline \multicolumn{2}{|c|}{ Skin rash/pruritus $(n=5)$} & $5(14.28 \%)$ & $2(5.71 \%)$ \\
\hline \multicolumn{2}{|l|}{ Pneumonitis $(n=3)$} & $3(8.57 \%)$ & $3(8.57 \%)$ \\
\hline \multicolumn{2}{|l|}{ Colitis $(n=2)$} & $2(5.71 \%)$ & $0(0 \%)$ \\
\hline \multicolumn{2}{|l|}{ Arthritis $(n=1)$} & $1(2.85 \%)$ & $0(0 \%)$ \\
\hline \multicolumn{2}{|l|}{ Transaminitis $(n=2)$} & $2(5.71 \%)$ & $0(0 \%)$ \\
\hline \multicolumn{2}{|l|}{ Myocarditis $(n=1)$} & $1(2.85 \%)$ & $1(2.85 \%)$ \\
\hline \multicolumn{2}{|l|}{ Retinopathy $(n=1)$} & $1(2.85 \%)$ & $0(0 \%)$ \\
\hline
\end{tabular}

Abbreviations: irAE, immune-related adverse event; mRCC, metastatic renal cell carcinoma. 
occurred was pneumonitis in three patients $(8.57 \%)$ and it occurred before 8 weeks in all of the three patients. Rare irAEs of myocarditis and immune mediated retinopathy were encountered in one patient each at 6 weeks and at 38 weeks, respectively. It was also observed that one case of skin rash and one case of colitis occurred as late as 36 and 44 weeks, respectively. Out of the 35 patients enrolled in the study, 6 patients $(17.14 \%)$ required systemic steroids (intravenous/oral steroids) for the management of irAEs. Additional immunosuppression in the form of mycophenolate mofetil (MMF) was used in one patient with Grade 3 pneumonitis. Treatment discontinuation due to irAEs occurred in three patients (8.57\%).

\section{Discussion}

Immune checkpoint inhibitors have led to a paradigm shift in the management of metastatic RCC. The landmark Checkmate025 trial established the role of PD-1 inhibitor nivolumab monotherapy in the setting of metastatic RCC that had progressed on first-line therapy. ${ }^{13}$ Currently, immunotherapy with PD1/PDL1 inhibitors is fast becoming the standard of care in the first-line setting in combination with VEGF TKI or dual checkpoint blockade with CTLA- 4 inhibitors. ${ }^{14,15}$ There, however, remains a paucity of data in the real-world setting especially in developing countries where access to Immune Checkpoint inhibitors (ICIs) remains low in view of cost and accessibility. This study was hence conducted to look at the efficacy and safety profile of nivolumab monotherapy in relapsed mRCC.

A majority of patients in our study, 30 (85.7\%) patients received nivolumab as second-line treatment for metastatic RCC following progression on first line. This pattern of usage is similar to that observed in Checkmate-025 trial and other real-world experience data from Spain where patients had received only one prior antiangiogenic therapy. ${ }^{16}$ Our study had an almost equal proportion of patients belonging to the IMDC intermediate and poor risk group and no patients in the good risk group. This was in stark contrast to the Checkmate-025 trial where a majority of patients belonged to the MSKCC good and intermediate risk group. ${ }^{13}$ Our study also differed from other real-world studies from France and Italy wherein majority of the patients belonged to the IMDC good and intermediate risk group. ${ }^{17,18}$

Despite these differences, nivolumab demonstrated high response rates in our study population with objective response rates of $60 \%$ being noted with CR being achieved by $11.4 \%$ of the patients. These response rates were higher than the response rates seen in the landmark Checkmate-025 trial (overall response rate [ORR]: $25 \%, \mathrm{CR}: 1 \%$ ) or other real-world studies from United Kingdom ( $n=109$, ORR: $31.5 \%)$ and France $(n=720$, ORR: $21 \%) .{ }^{17,19}$ Apart from a study with larger sample size, further research would need to be performed to analyze for probable difference in genetic signatures that may be accounting for the higher response rates in our population. More than half of the responders in our study have a duration of response lasting beyond a year as compared with $31 \%$ of those in the Checkmate- 025 trial who had a response duration lasting for more than a year.
The median PFS in our study was 5 months that again was similar to the PFS of 4.6 months seen in the Checkmate-025 trial. ${ }^{13}$ This trend is seen in the real-world studies in Italy, United Kingdom, Cleveland, where the median PFS was found to be $4.5,5.4$, and 4.8 months, respectively. ${ }^{18-20}$ Among the five patients who were treated beyond progression in our study, three went onto achieve further reduction and demonstrated prolonged survival trends. The clinical benefit of a decrease in tumor burden with an increase in OS in a proportion of patients who were treated beyond progression with nivolumab as compared with those not treated beyond progression was also reported by Escudier et al in the Checkmate-025 study. ${ }^{21}$

Similar to the landmark Checkmate-025 trial, ${ }^{13}$ the median OS in our study was 26.0 months. Ongoing survival of 47 , 42,34 , and 22 months was noted in four patients who had achieved CR. These patients had stopped treatment after a duration of 1 year and continue to be in complete remission. A similar median OS of 22.1 months was observed in the IMDC analysis of real-world outcomes with nivolumab in the treatment of clear cell RCC in second line or beyond. ${ }^{22}$ In our study, the survival between responders and nonresponders was statistically significantly different with the median OS not reached in responders and was only 4 months in nonresponders $(p<0.001)$. This highlights that those who are responders go on to have a prolonged survival. While there is scarcity of literature on the difference in survival between responders and nonresponders in metastatic RCC, a pooled analysis of the various trials of nivolumab in previously treated advanced/metastatic NSCLC showed that responders have a significantly prolonged survival as compared with non-responders. ${ }^{23}$ It was also noted in our study that there was no statistically significant difference in the survival between the IMDC intermediate-risk and poor-risk patients. This was in contrast to the NIVOREN GETUG AFU 26 realworld study from France where the poor-risk group demonstrated a median OS of only 10.4 months as compared with 25.0 and 32.8 months, respectively, in the intermediate- and favorable-risk patients. ${ }^{17}$

The question of when to stop immunotherapy in patients with metastatic disease after achieving response is being explored in the scientific field. Four patients in our study who achieved CR stopped treatment with nivolumab after having received it for a duration of 1 year highlighting the durable and long-lasting response of immunotherapy. These findings are also reflected in the data from Keynote-001 trial that showed that $94 \%$ (63 of 67) of metastatic melanoma patients who achieved a CR on pembrolizumab and did not receive further subsequent anticancer therapy remained disease free for 30 months after stopping treatment. ${ }^{24}$ While data from a Phase- 2 trial by Ornstein et al showed promising results for the role of intermittent nivolumab in $\mathrm{mRCC},{ }^{25}$ further studies need to be done to evaluate the optimal timing of stopping therapy after obtaining CR or the maximal reduction in tumor burden.

In our study, the adverse events reported were predominantly irAEs. Twenty-three patients (65.7\%) experienced any grade of IrAE, while four patients (11.42\%) experienced Grade 3 
irAEs. This safety profile was consistent with the Checkmate025 trial wherein 19\% developed Grade 3 treatment-related adverse effect. ${ }^{13}$ Real-world studies from France and United Kingdom have reported similar safety profile with respect to Grade 3 adverse events (17.3 and 16.9\%, respectively). ${ }^{17,19}$ Thyroid dysfunction was the most common irAE, followed by fatigue in our study. Rare toxicities of immune-related myocarditis and immune-mediated retinopathy were also seen in the study. The spectrum of irAE seen in our setting was different from real-world studies in MRCC treated with nivolumab from Italy and Japan that showed cutaneous irAE to be the most common irAE followed by endocrinological and hepatobiliary irAEs ${ }^{18,26}$. It is also interesting to note that none of the patients in our study or in other real-world studies from Italy reported adverse events such as nausea, stomatitis, and dysgeusia that were reported in more than $10 \%$ of patients in the Checkmate-025 trial.

The timing of onset of various irAEs after initiation of treatment with nivolumab was consistent with what has been described in literature with median onset of thyroid dysfunction in our study being 10 weeks and cutaneous irAEs occurring earlier at a median of 6 weeks since initiation of treatment. ${ }^{27-29}$ Delayed onset of irAEs was also observed as late as after 30 weeks in some cases. Hence, it is imperative to be aware of the most common time of occurrence of irAEs and also to remember that it can be unpredictable and occur at any point of time even after stoppage of treatment as it is noted from literature that the first irAE can even occur as long as 1 year after discontinuation of treatment. ${ }^{28}$

Steroids were the cornerstone for the management of irAE with additional immunosuppression with MMF being required in only one patient. No patient of hyperthyroidism required antithyroid drugs as they subsequently went onto become euthyroid/hypothyroid. All patients with hypothyroidism received thyroid supplements eventually. Treatment discontinuation due to irAE occurred in three patients ( $8.57 \%$ ). This is similar to multiple studies including the Checkmate 025 study and NIVOREN GETUG AFU 26 study where treatment discontinuation due to adverse events occurred in 8 and $7.5 \%$ of patients, respectively. ${ }^{13,17}$

\section{Conclusion}

Nivolumab demonstrated good efficacy with objective response being noted in $60 \%$ of our patients. The OS trend noticed in our population is similar to the landmark Checkmate-025 trial and other real-world studies, despite most of the patients belonging to the IMDC intermediate- and poorrisk group. Nivolumab was well tolerated with safety profile in terms of irAE being consistent with those described in literature with less than $10 \%$ requiring discontinuation of treatment due to irAEs.

We acknowledge that the first-line approach in $\mathrm{MRCC}$ is rapidly changing to immunotherapy-based combinations throughout the world. But due to the financial implications, oral sunitinib or pazopanib will continue to be extensively used in India as first-line therapy. So, we believe immuno- therapy with nivolumab will have an important role to play as second-line treatment in $\mathrm{MRCC}$ in India.

\section{Authors' Contribution}

Dr. Amit Rauthan was involved in conceptualization of study design, data Collection and analysis, and preparation of manuscript.

Dr. Nitin Yashas Murthy was involved in conceptualization of study design, data collection and analysis, and preparation of manuscript.

Dr. Poonam Patil conceptualized study design, reviewed and edited the manuscript.

Dr. Gaurav Nigade was involved in data collection and analysis and preparation of manuscript.

Dr. Somashekhar SP conceptualized study design, reviewed and edited the manuscript.

Dr. Shabber Zaveri conceptualized study design, reviewed and edited the manuscript.

The final manuscript has been read and approved by all the authors. The revision of the manuscript based on the recommendations of the editors/reviewers has been incorporated and also been read and approved by all the authors.

\section{Disclosures}

As mentioned in the ICJME Disclosure form:

Dr. Amit Rauthan has received honoraria for lectures/presentations/educational events from Bristol Myers Squibb, MSD, and Roche. Rest of the authors have no disclosures to be made.

\section{Conflict of Interest}

None declared.

\section{References}

1 Hsieh JJ, Purdue MP, Signoretti S, et al. Renal cell carcinoma. Nat Rev Dis Primers 2017;3:17009

2 Ferlay J, Ervik M, Lam F, Colombet M, Mery L, Piñeros M, Znaor A, Soerjomataram I, Bray F. Global Cancer Observatory: Cancer Today. Lyon, France: International Agency for Research on Cancer [Internet] 2020 [cited 2021 Apr 10]. Accessed September 30, 2021 from: https://gco.iarc.fr/today

3 Motzer RJ, Hutson TE, Tomczak P, et al. Sunitinib versus interferon alfa in metastatic renal-cell carcinoma. N Engl J Med 2007;356 (02):115-124

4 Sternberg CN, Davis ID, Mardiak J, et al. Pazopanib in locally advanced or metastatic renal cell carcinoma: results of a randomized phase III trial. J Clin Oncol 2010;28(06):1061-1068

5 Escudier B, Eisen T, Stadler WM, et al; TARGET Study Group. Sorafenib in advanced clear-cell renal-cell carcinoma. N Engl J Med 2007;356(02):125-134

6 Rini BI, Escudier B, Tomczak P, et al. Comparative effectiveness of axitinib versus sorafenib in advanced renal cell carcinoma (AXIS): a randomised phase 3 trial. Lancet 2011;378(9807):1931-1939

7 Motzer RJ, Escudier B, Oudard S, et al; RECORD - 1 Study Group. Phase 3 trial of everolimus for metastatic renal cell carcinoma: final results and analysis of prognostic factors. Cancer 2010;116 (18):4256-4265

8 Thompson RH, Kuntz SM, Leibovich BC, et al. Tumor B7-H1 is associated with poor prognosis in renal cell carcinoma patients with long-term follow-up. Cancer Res 2006;66(07):3381-3385 
9 Brahmer JR, Drake CG, Wollner I, et al. Phase I study of singleagent anti-programmed death-1 (MDX-1106) in refractory solid tumors: safety, clinical activity, pharmacodynamics, and immunologic correlates. J Clin Oncol 2010;28(19):3167-3175

10 Topalian SL, Hodi FS, Brahmer JR, et al. Safety, activity, and immune correlates of anti-PD-1 antibody in cancer. $\mathrm{N}$ Engl J Med 2012;366(26):2443-2454

11 Choueiri TK, Fishman MN, Escudier B, et al. Immunomodulatory activity of nivolumab in metastatic renal cell carcinoma. Clin Cancer Res 2016;22(22):5461-5471

12 Motzer RJ, Rini BI, McDermott DF, et al. Nivolumab for metastatic renal cell carcinoma: results of a randomized phase II trial. J Clin Oncol 2015;33(13):1430-1437

13 Motzer RJ, Escudier B, McDermott DF, et al; CheckMate 025 Investigators. Nivolumab versus everolimus in advanced renalcell carcinoma. N Engl J Med 2015;373(19):1803-1813

14 Rini BI, Plimack ER, Stus V, et al; KEYNOTE-426 Investigators. Pembrolizumab plus axitinib versus sunitinib for advanced renalcell carcinoma. N Engl J Med 2019;380(12):1116-1127

15 Motzer RJ, Tannir NM, McDermott DF, et al; CheckMate 214 Investigators. Nivolumab plus ipilimumab versus sunitinib in advanced renal-cell carcinoma. N Engl J Med 2018;378(14):1277-1290

16 Amores Bermúdez J, Osman García I, Unda Urzáiz M, et al. Safety of nivolumab in metastatic renal cell carcinoma patients: a real-life experience in a Spanish urology setting. Actas Urol Esp (Engl Ed) 2019;43(07):364-370(English Edition)

17 Albiges L, Negrier S, Dalban C, et al. Safety and efficacy of nivolumab in metastatic renal cell carcinoma (mRCC): final analysis from the NIVOREN GETUG AFU 26 study. J Clin Oncol 2019;37:542-542

18 Verzoni E, Cartenì G, Cortesi E, et al; Italian Nivolumab Renal Cell Cancer Early Access Program group. Real-world efficacy and safety of nivolumab in previously-treated metastatic renal cell carcinoma, and association between immune-related adverse events and survival: the Italian expanded access program. J Immunother Cancer 2019;7(01):99

19 Hack J, Hill SL, Broadfoot J, et al. Real world experience of nivolumab therapy in metastatic renal cancer patients: a 3 year multi-centre review. Ann Oncol 2019;30:v389
20 Zahoor H, Barata PC, Jia X, et al. Patterns, predictors and subsequent outcomes of disease progression in metastatic renal cell carcinoma patients treated with nivolumab. J Immunother Cancer 2018;6(01):107

21 Escudier B, Motzer RJ, Sharma P, et al. Treatment beyond progression in patients with advanced renal cell carcinoma treated with nivolumab in CheckMate 025. Eur Urol 2017;72(03):368-376

22 Stukalin I, Wells JC, Graham J, et al. Real-world outcomes of nivolumab and cabozantinib in metastatic renal cell carcinoma: results from the International Metastatic Renal Cell Carcinoma Database Consortium. Curr Oncol 2019;26(02):e175-e179

23 Antonia SJ, Borghaei H, Ramalingam SS, et al. Four-year survival with nivolumab in patients with previously treated advanced non-small-cell lung cancer: a pooled analysis. Lancet Oncol 2019; 20(10):1395-1408

24 Betof Warner A, Palmer JS, Shoushtari AN, et al. Long-term outcomes and responses to retreatment in patients with melanoma treated with PD-1 blockade. J Clin Oncol 2020;38(15):1655-1663

25 Ornstein MC, Wood LS, Hobbs BP, et al. A phase II trial of intermittent nivolumab in patients with metastatic renal cell carcinoma (mRCC) who have received prior anti-angiogenic therapy. J Immunother Cancer 2019;7(01):127

26 Numakura K, Kobayashi M, Hatakeyama S, et al. Efficacy and safety of nivolumab for renal cell carcinoma in patients over 75 years old from multiple Japanese institutes. Int J Clin Oncol 2020;25(08):1543-1550

27 Lee H, Hodi FS, Giobbie-Hurder A, et al. Characterization of thyroid disorders in patients receiving immune checkpoint inhibition therapy. Cancer Immunol Res 2017;5(12):1133-1140

28 Haanen JBAG, Carbonnel F, Robert C, et al; ESMO Guidelines Committee. Management of toxicities from immunotherapy: ESMO Clinical Practice Guidelines for diagnosis, treatment and follow-up. Ann Oncol 2017;28(suppl_4):iv119-iv142

29 Puzanov I, Diab A, Abdallah K, et al; Society for Immunotherapy of Cancer Toxicity Management Working Group. Managing toxicities associated with immune checkpoint inhibitors: consensus recommendations from the Society for Immunotherapy of Cancer (SITC) Toxicity Management Working Group. J Immunother Cancer 2017;5(01):95 